Canum 2000: Actes du 32e Congrès national d'analyse numérique

A. Blouza, I. Danaila, P. Joly, S.M. Kaber, B. Lucquin, F. Murat \& M. Postel, Éditeurs

ESAIM: Proceedings, Vol. 11, 2002, 127-140

http://www.emath.fr/Maths/Proc/Vol.11/

(C)2002, Société de Mathématiques Appliquées et Industrielles, EDP Sciences

\title{
Problématiques numériques pour la simulation moléculaire
}

\author{
Claude Le Bris
}

\begin{abstract}
Résumé. L'objet de l'exposé est de faire un rapide état de l'art des connaissances d'analyse numérique sur les modèles de la simulation moléculaire, en mettant l'accent sur quelques avancées nouvelles. On détaillera quelques résultats récents (obtenus en collaboration avec E. Cancès) sur l'analyse numérique des algorithmes existants et sur les performances d'algorithmes nouveaux. La problématique générale est à la fois celle de l'optimisation numérique en grande dimension, et celle de la résolution numérique de problèmes aux valeurs propres non linéaires. On donnera ensuite un court aperçu de travaux en cours sur le contrôle des réactions chimiques par laser. Des techniques de différentiation automatique y sont en particulier mises en oeuvre (travaux avec A. Ben-Haj-Yedder).
\end{abstract}

Mots clés. Optimisation sous contraintes, problèmes aux valeurs propres non linéaires, champ moyen, équations de Hartree-Fock, algorithmes numériques, contrôle optimal, équation de Schrödinger, différentiation automatique

\begin{abstract}
We present a brief overview of the state of the art of the numerical techniques in use for the simulation of molecular systems in the framework of quantum chemistry. We focus on the simulation of "small" systems and we lay some emphasis on some recent methods. The issue of the optimal control of chemical reactions using laser fields is also discussed
\end{abstract}

Key words. constrained optimization, nonlinear eigenvalue problem, mean field theory, Hartree-Fock equations, numerical algorithms, optimal control, Schrödinger equation, automatic differentiation

AMS subject classification. 65B, 65M, 65N, 81V55

\section{Introduction aux modèles de la simulation moléculaire}

La simulation moléculaire a pour but de comprendre et prévoir les propriétés macroscopiques d'une substance à partir de la simulation de ses constituants à l'échelle microscopique. Typiquement, on peut distinguer trois grandes classes de modèles

1. les modèles dits $a b$ initio, basés uniquement sur la mise en équation des principes de la mécanique quantique (voir [16, 21, 26, 29]), 
2. les modèles dits semi-empiriques, qui sont des simplifications des précédents en ce qu'ils négligent certains termes jugés "secondaires" (comme des interactions à longue distance par exemple), et court-circuitent certains calculs trop longs en les remplaçant par des valeurs tabulées, expérimentales au besoin,

3. les modèles de la dynamique moléculaire et assimilés, dans lesquels on quitte majoritairement la mécanique quantique pour la remplacer par la mécanique classique newtonienne (voir $[18,14]$ ).

Evidemment, l'applicabilité de ces modèles varie de plusieurs ordres de grandeur : un modèle $a b$ initio est aujourd'hui limité à la simulation de systèmes disons d'une centaine d'atomes, alors que par dynamique moléculaire on peut simuler convenablement des systèmes de plusieurs millions d'atomes. Le sens de l'Histoire est évidemment d'aller de plus en plus vers la simulation de gros systèmes : un virus compte typiquement des millions d'atomes (voir par exemple [31] pour la simulation en biologie). Pourtant, nous allons nous limiter dans cet exposé au cas des modélisations ab initio. Pourquoi ?

1. d'abord parce que les petits systèmes auxquels ces modèles sont dédiés restent encore aujourd'hui d'un grand intérêt en eux-mêmes : ils sont encore loin d'être tous connus parfaitement.

2. ensuite parce qu'ils sont le cadre idéal pour les études précises d'analyse numérique et les tests de nouvelles stratégies numériques,

3. enfin parce que ces modèles bien que limités en taille sont aussi d'applicabilité grande. Ils viennent en effet très souvent "irriguer" les modèles plus grossiers, et ce au moins de deux façons. Dans les gros systèmes d'intérêt pratique, certes la majeure partie du système peut la plupart du temps être simulée par dynamique moléculaire car elle est, pour une application donnée, "inerte chimiquement", mais elle comporte aussi souvent un "petit" site actif qui subit la réaction chimique intéressante : ce dernier sera donc, lui, modélisé ab initio. On parle alors de modélisations QM/MM pour Quantum Mechanics/Molecular Mechanics. Dans le même esprit, les calculs précis ab initio sur les petits systèmes servent à définir ("fitter") les champs de force interatomiques qui seront utilisés dans la dynamique newtonienne.

Nous ne développerons pas tous les aspects théoriques (ils sont nombreux) liés aux modèles de la chimie quantique. Nous renvoyons pour cela à $[5,11,12,13,23]$ et leurs bibliographies. En vertu de ce qui a été dit ci-dessus, nous nous concentrons sur la simulation numérique d'un petit système moléculaire. Expliquons brièvement de quoi il est question.

Le coeur d'un code de chimie quantique est constitué du calcul de l'état fondamental électronique du système moléculaire à configuration de noyaux donnée (les $\left.\bar{x}_{k}\right)$. Il s'agit idéalement du problème de minimisation

$$
\inf \left\{<H_{N} \Psi, \Psi>, \Psi \in L^{2}\left(\mathbf{R}^{3 N}\right), \Psi \text { est antisymétrique, } \int_{\mathbf{R}^{3 N}}|\Psi|^{2}=1\right\},
$$

ESAIM: Proc., Vol. 11, 2002, 127-140 
où $H_{N}$ est le Hamiltonien à $N$ corps

$$
H_{N}=-\sum_{i=1}^{N}\left(\Delta_{x_{i}}+V\left(x_{i}\right)\right)+\frac{1}{2} \sum_{i \neq j} \frac{1}{\left|x_{i}-x_{j}\right|}
$$

et $V$ le potentiel d'attraction créé par les noyaux $V(x)=-\sum_{k=1}^{K} \frac{z_{k}}{\left|x-\bar{x}_{k}\right|}$.

Comme ce problème n'est toujours pas à l'heure actuelle attaquable tel quel numériquement, on en fait une approximation, et pour cet exposé introductif, nous nous contenterons de décrire l'approximation dite de Hartree-Fock de ce problème (une autre catégorie d'approximations, comme celle de la fonctionnelle de la densité [16], conduirait sensiblement aux mêmes problématiques numériques). Dans l'approximation de Hartree-Fock, on remplace les fonctions tests $\Psi$ générales par celles qui sont un déterminant de $N$ fonctions $L^{2}\left(\mathbf{R}^{3}\right)$. Le prix à payer pour avoir alors rendu (1) traitable numériquement est d'avoir à traiter un problème de minimisation non quadratique

$$
\begin{array}{r}
I^{H F}=\inf \left\{\sum_{i=1}^{N} \int_{\mathbf{R}^{3}}\left(\left|\nabla \Phi_{i}\right|^{2}+V\left|\Phi_{i}\right|^{2}\right)+\frac{1}{2} \iint_{\mathbf{R}^{3} \times \mathbf{R}^{3}} \frac{\left|\Phi_{i}(x) \Phi_{j}(y)-\Phi_{j}(x) \Phi_{i}(y)\right|^{2}}{|x-y|}\right. \\
\left.\Phi_{i} \in H^{1}\left(\mathbf{R}^{3}\right), \int_{\mathbf{R}^{3}} \Phi_{i} \Phi_{j}^{\star}=\delta_{i j}\right\}
\end{array}
$$

ce qui revient à dire que son équation d'Euler-Lagrange est une EDP hautement non linéaire, à savoir le système non linéaire aux valeurs propres suivant

(4) $-\Delta \Phi_{i}+V \Phi_{i}+\left(\sum_{j=1}^{N}\left|\Phi_{j}\right|^{2} \star \frac{1}{|x|}\right) \Phi_{i}-\sum_{j=1}^{N}\left(\Phi_{i} \Phi_{j}^{\star} \frac{1}{|x|}\right) \Phi_{j}=-\varepsilon_{i} \Phi_{i}, i=1, \ldots, N$.

qui peut se mettre, avec des notations évidentes, sous la forme condensée

$$
H_{\Phi} \Phi_{i}=-\varepsilon_{i} \Phi_{i}
$$

dite équation SCF pour "self-consistent field".

L'essentiel des méthodes numériques s'attache à résoudre le problème via la résolution des équations (5), et non via une attaque directe du problème de minimisation (3). Après avoir discrétisé ces équations (on met en oeuvre une méthode type Galerkin - dite LCAO en chimie, pour Linear combination of Atomic Orbitals - en développant sur une base de fonctions propres du Hamiltonien des atomes isolés, ce qui pose d'ailleurs d'intéressantes questions sur la complétude des bases employées, sur la convergence de la solution discrétisée vers la solution exacte, et sur l'estimation de l'erreur commise, voir sur ces sujets les études menées par Y. Maday et G. Turinici [24]), on fait une résolution self-consistante de ces équations.

Il n'est pas inutile ici de dire quelques mots sur la complexité algorithmique d'une telle résolution. Comme on peut le voir sur la forme (4) en faisant en pensée la formulation faible, la complexité de la phase d'assemblage de la matrice associée au membre de gauche (dite matrice de 
Fock) est en fait $N^{4}, N$ désignant la taille de la base de discrétisation. Cette phase d'assemblage domine donc en complexité les autres phases, et en particulier la diagonalisation. Une telle complexité est due aux termes de convolution dans (4), où figure le potentiel coulombien (donc longue distance) qui couple tous les éléments entre eux. Cette complexité serait un peu plus faible pour une méthode DFT ( $N^{3}$ ou un peu moins), mais bien plus grande pour des méthodes dites postHartree-Fock : jusqu'à $N^{8}$ pour des méthodes multi-configurations qui consistent à prendre pour $\Psi$ dans (1) des sommes de déterminants au lieu d'un seul. Toutes les méthodes ab initio ont donc un scaling du type $k N^{p}$ avec $p=3,4,5, \ldots, 8$. Comme on l'imagine aisément, ce scaling est un vrai problème pratique. En effet, il s'agit de bien garder à l'esprit que ce problème de minimisation (3) n'est pas uniquement un préalable à un calcul plus complet. Il peut être aussi une étape d'un calcul d'optimisation de géométrie (il y une boucle externe en les positions $\bar{x}_{k}$ des noyaux qu'on optimise pour atteindre la configuration énergétiquement la plus stable), ou un pas de temps d'une simulation dépendante du temps dans l'approximation adiabatique, où à chaque nouveau jeu de positions de noyaux on calcule l'état électronique stationnaire du système. Cela donne quelque chose du genre :

$$
\left\{\begin{array}{l}
m_{k} \frac{d^{2} \bar{x}_{k}}{d t^{2}}(t)=-\nabla_{\bar{x}_{k}}\left(U\left(\bar{x}_{1}(t), \cdots, \bar{x}_{K}(t)\right)+\sum_{1 \leq l<m \leq K} \frac{z_{l} z_{m}}{\left|\bar{x}_{l}-\bar{x}_{m}\right|}\right) \\
U\left(\bar{x}_{1}, \cdots, \bar{x}_{K}\right)=\inf \left\{\left\langle\Psi, H_{N}\left(\bar{x}_{1}, \cdots, \bar{x}_{K}\right) \cdot \Psi\right\rangle, \quad \Psi\right\}
\end{array}\right.
$$

Ces deux seuls cas suffisent à faire comprendre qu'on peut avoir à résoudre (1) (ou (5)) en un temps record, alors qu'une seule fois est déjà un challenge! Penser qu'une simulation sur une nanoseconde d'un système moléculaire (ce qui est un temps ridiculement petit) requiert un milion de pas de temps sur la dynamique des noyaux (le temps caractéristique de leur vibration est $10^{-15} \mathrm{~s}$ ), donc la résolution d'un million de problèmes de type (1) .... Motiver la nécessité de méthodes rapides pour de tels problèmes est un jeu d'enfant !

Longtemps, l'effort n'a été porté que sur la diminution du préfacteur $k$ (abaissé de 6 ordres de grandeur en 15 ans), mais pas sur l'ordre de complexité $p$ des méthodes. Ce n'est que très récemment qu'on a vu émerger des méthodes dites $O(N)$ ("linear scaling"), si possible atteignant très vite cette limite asymptotique et avec un bon préfacteur. Ces dernières sont le plus souvent basées à la fois sur un assemblage plus rapide de la matrice de Fock (méthodes fast multipole, ...) et sur des techniques visant soit à effectuer très vite sa diagonalisation, soit à l'éviter en revenant à des approches plus variationnelles (density matrix methods, ...). On ne traitera pas ici le sujet des méthodes $O(N)$, pour lequel on renvoie par exemple à [10,19,20] et aussi à [25] pour les aspects parallélisme.

Compte tenu de l'intérêt souligné ci-dessus du calcul rapide et précis de petites molécules, nous préférons nous cantonner au cas des méthodes "classiques" consistant à résoudre (5) (sans compter qu'il n'est pas clair que les méthodes $O(N)$ développées à ce jour s'appliquent dans toutes les situations : un contrexemple célèbre est celui des métaux, ce qui est en fait mathématiquement lié à une mauvaise "séparation" des valeurs propres dans le spectre de l'opérateur de Fock, et met en échec les techniques habituelles d'accélération). Plus précisément, nous nous intéressons à l'itération sur la non linéarité considérant (pour l'instant...) la phase linéaire de diagonalisation à chaque itération comme une boite noire supposée optimisée par ailleurs.

ESAIM: Proc., Vol. 11, 2002, 127-140 
Une analyse numérique sérieuse des méthodes qui sont employées depuis plusieurs années dans ce contexte n'a débuté que récemment. Comme on le verra (Section 3), une telle analyse a permis de proposer des algorithmes plus rigoureusement fondés, ayant donc des chances raisonnables de mieux fonctionner génériquement, et qui sont actuellement en cours de tests. Il n'est pas exclus de penser qu'une fois ces algorithmes mis au point, ils pourront efficacement s'interfacer avec, ou s'inclure dans, des approches $O(N)$.

\section{Analyse numérique des algorithmes SCF}

Cette section et la suivante décrivent un travail en collaboration avec E. Cancès. Les détails peuvent être lus dans $[4,6,7,8]$.

Pour formaliser convenablement l'analyse numérique des algorithmes SCF, le bon langage est celui des matrices densité. Rappelons qu'à toute fonction test Hartree-Fock $\Phi:=\left(\Phi_{1}, \ldots, \Phi_{N}\right)$ dans (3), on peut associer la matrice densité

$$
\mathcal{D}_{\Phi}=\sum_{i=1}^{N}\left(\Phi_{i}, \cdot\right)_{L^{2}\left(\mathbf{R}^{3}\right)} \Phi_{i},
$$

la matrice densité réduite $\tau_{\mathcal{D}_{\Phi}}(x, y)=\sum_{i=1}^{N} \Phi_{i}(x) \Phi_{i}^{\star}(y)$ et la densité $\rho_{\mathcal{D}_{\Phi}}(x)=\tau_{\mathcal{D}_{\Phi}}(x, x)$. Le problème Hartree-Fock se récrit alors

$$
\begin{aligned}
I^{H F}= & \inf \left\{\mathcal{E}^{H F}(\mathcal{D}), \operatorname{Tr}(\mathcal{D})=N,, \mathcal{D}^{2}=\mathcal{D}=\mathcal{D}^{\star}\right\} \\
& \mathcal{E}^{H F}(\mathcal{D}):=\operatorname{Tr}(h \mathcal{D})+\frac{1}{2} \operatorname{Tr}(\mathcal{G}(\mathcal{D}) \cdot \mathcal{D})
\end{aligned}
$$

avec $h:=-\Delta+V$ et

$$
\mathcal{G}(\mathcal{D}) . \Phi:=\left(\rho_{\mathcal{D}} \star \frac{1}{|x|}\right) \Phi-\int_{\mathbf{R}^{3}} \frac{\tau_{\mathcal{D}}(x, y)}{|x-y|} \Phi(y) d y .
$$

En utilisant l'opérateur de Fock

$$
\mathcal{F}(\mathcal{D}):=h+\mathcal{G}(\mathcal{D})
$$

les équations de Hartree-Fock (4) se récrivent

$$
\mathcal{F}(\mathcal{D}) \Phi_{i}=-\varepsilon_{i} \Phi_{i}
$$

et il s'agit en fait (en vertu d'un résultat théorique) de trouver les $N$ plus petites fonctions propres de l'opérateur.

La façon la plus standard de résoudre (12) est un algorithme de point fixe (connu sous le nom d'algorithme de Roothaan)

$$
\mathcal{D}_{n} \longrightarrow \text { assembler } \mathcal{F}\left(\mathcal{D}_{n}\right) \longrightarrow \text { diagonaliser } \mathcal{F}\left(\mathcal{D}_{n}\right) \stackrel{\text { aufbau }}{\longrightarrow} \mathcal{D}_{n+1}
$$


où aufbau désigne le processus consistant à prendre les $N$ plus petites fonctions propres. Tel quel, cet algorithme n'est quasiment plus utilisé. On a récemment expliqué, par une analyse mathématique complète de cet algorithme, les raisons pour lesquelles il exhibe le comportement suivant : soit il converge, soit il oscille entre deux états dont aucun n'est solution du problème.

Un bien meilleur algorithme est celui connu sous le nom d'algorithme Direct Inversion in the Iterated Subspace (DIIS) [27, 28]. En fait, il exploite la propriété suivante : il y a équivalence entre être solution de (12) et vérifier la relation de commutation $[\mathcal{F}(\mathcal{D}), \mathcal{D}]=0$. L'idée est de procéder comme suit :

Etape 1 Faire $p$ itérations de l'algorithme de Roothaan (13) en gardant en mémoire les matrices $\mathcal{D}_{1}, \mathcal{D}_{2}, \ldots, \mathcal{D}_{p}$

Etape 2 Définir les coefficients $\left(\bar{c}_{1}, \ldots, \bar{c}_{p}\right) \in \mathbf{R}^{p}$ comme les minimiseurs de

$$
\inf \left\{\left\|\sum_{i=1}^{p} c_{i}\left[\mathcal{F}\left(\mathcal{D}_{i}\right), \mathcal{D}_{i}\right]\right\| ; \sum_{i=1}^{p} c_{i}=1,0 \leq c_{i} \leq 1\right\}
$$

Etape 3 Assembler la matrice $\overline{\mathcal{F}}=\sum_{i=1}^{p} \bar{c}_{i} \mathcal{F}_{i}$

Etape 4 Diagonaliser $\overline{\mathcal{F}}$ et assembler $\mathcal{D}_{p+1}$ par aufbau

Etape 5 Ajouter $\mathcal{D}_{p+1}$ à la liste des matrices et retourner à l'Etape 2

Il s'avère que cet algorithme marche extrèmement bien, surtout si on le couple à la technique de level-shifting (aussi étudiée rigoureusement dans [6]) consistant à remplacer $\mathcal{F}\left(\mathcal{D}_{n}\right)$ par $\mathcal{F}_{n}^{b}=$ $\mathcal{F}\left(\mathcal{D}_{n}\right)-b \mathcal{D}_{n}$ (pour une constante $b$ assez grande). Malheureusement, il existe encore des cas où cet algorithme ne converge pas, et de plus on manque actuellement d'une analyse numérique de cet algorithme. C'est une question ouverte de savoir pourquoi il converge quand il converge.

Ceci nous a motivé pour développer une approche meilleure, en ce qu'on peut prouver effectivement sa convergence, et qu'on observe en pratique une convergence essentiellement plus rapide.

\section{Un nouvel algorithme}

L'idée de base est de s'assurer que l'énergie de Hartree-Fock décroît effectivement au cours des itérations (ce qui n'est pas le cas dans DIIS). Nous remplaçons donc dans l'Etape 2 de l'algorithme DIIS la relaxation sur les commutateurs par une relaxation fondée sur la minimisation sur un espace de petite dimension de la fonctionnelle de Hartree-Fock elle-même. Ceci un peu dans l'esprit de [3].

A des points techniques près qu'on ne détaille pas ici, on obtient ainsi l'algorithme :

Etape 1 Supposons qu'on dispose de $p$ matrices densité $\mathcal{D}_{1}, \mathcal{D}_{2}, \ldots, \mathcal{D}_{p}$ bâties lors des $p$ itérations précédentes, et qu'on n'a pas encore convergé. Le nombre $p$ est typiquement petit. 
Etape 2 On remarque que l'énergie de Hartree-Fock de $\mathcal{D}=\sum_{i=1}^{p} \lambda_{i} \mathcal{D}_{i}, \lambda_{i} \in[0,1], \sum_{i=1}^{p} \lambda_{i}=1$ est une fonction simple des coefficients $\lambda_{i}$ et qu'on peut donc trouver son minimiseur $\left(\tilde{\lambda}_{1}, \ldots, \tilde{\lambda}_{p}\right)$. On note $\widetilde{\mathcal{D}_{p}}$, laquelle n'est pas forcément un projecteur : $\widetilde{\mathcal{D}_{p}}{ }^{2} \neq \widetilde{\mathcal{D}_{p}}$ en général.

Etape 3 On veut déterminer à partir de $\widetilde{\mathcal{D}_{p}}$ une matrice densité vérifiant les contraintes de (8). On développe donc l'énergie (9) au voisinage de $\widetilde{\mathcal{D}_{p}}$ dans une direction $d$ :

$$
\mathcal{E}^{H F}\left(\widetilde{\mathcal{D}_{p}}+\mu d\right)=\mathcal{E}^{H F}\left(\widetilde{\mathcal{D}_{p}}\right)+\mu \operatorname{Tr}\left(\mathcal{F}\left(\widetilde{\mathcal{D}_{p}}\right) \cdot d\right)+\frac{\mu^{2}}{2} \operatorname{Tr}(\mathcal{G}(d) \cdot d) .
$$

Et on choisit alors la direction "steepest descent". On sait bien qu'elle n'est pas forcément la meilleure en toute généralité, mais elle est particulièrement facile à déterminer (ce qui n'est pas le cas des autres...). En effet, on remarque que

$$
\inf \left\{\operatorname{Tr}\left(\mathcal{F}\left(\widetilde{\mathcal{D}_{p}}\right) \cdot \mathcal{D}\right), \operatorname{Tr}(\mathcal{D})=N, \mathcal{D}^{2}=\mathcal{D}=\mathcal{D}^{\star}\right\}
$$

est atteint en la matrice $\mathcal{D}_{p+1}$ constituée des plus petites fonctions propres de $\mathcal{F}\left(\widetilde{\mathcal{D}_{p}}\right)$, et donc précisément déterminée par la solution de (12). Si on n'a pas convergé, on peut alors montrer que

$$
\operatorname{Tr}\left(\mathcal{F}\left(\widetilde{\mathcal{D}_{p}}\right) \cdot\left(\mathcal{D}_{p+1}-\widetilde{\mathcal{D}_{p}}\right)\right)<0,
$$

et donc la direction $d=\mathcal{D}_{p+1}-\widetilde{\mathcal{D}_{p}}$ est la direction de descente "la plus profonde" au sens de (16).

Etape 4 On ajoute $\mathcal{D}_{p+1}$ à la liste des matrices densité, et on retourne à l'Etape 2.

Cet algorithme assure la décroissance de l'énergie Hartree-Fock en passant de $\widetilde{\mathcal{D}_{p}}$ à $\widetilde{\mathcal{D}_{p+1}}$. Et on remarque ensuite (tout ceci se mathématise...) que peu à peu, $\widetilde{\mathcal{D}_{p}}$ se rapproche de l'ensemble des projecteurs. D'où la convergence. Dans la pratique, cet algorithme n'est pas exactement programmé sous la version indiquée ci-dessus, mais l'esprit reste.

Il est bon de remarquer que cet algorithme, meilleur que DIIS à la fois sur le plan théorique et sur le plan pratique, n'est guère plus coûteux. Il s'agit seulement d'ajouter une minimisation en les $\lambda_{i}$, ce qui ne se connaît pas en termes de temps calcul. Nous travaillons actuellement sur des versions au second ordre de cet algorithme.

\section{Vers le contrôle par laser des réactions chimiques}

La section précédente décrivait des travaux sur la simulation stationnaire, avec cette idée déjà mentionnée qu'elle n'est peut être qu'un pas de temps d'une simulation dynamique.

Donnons dans cette dernière section un exemple de problématique typiquement liée à la simulation des phénomènes dépendants du temps.

ESAIM: Proc., Vol. 11, 2002, 127-140 

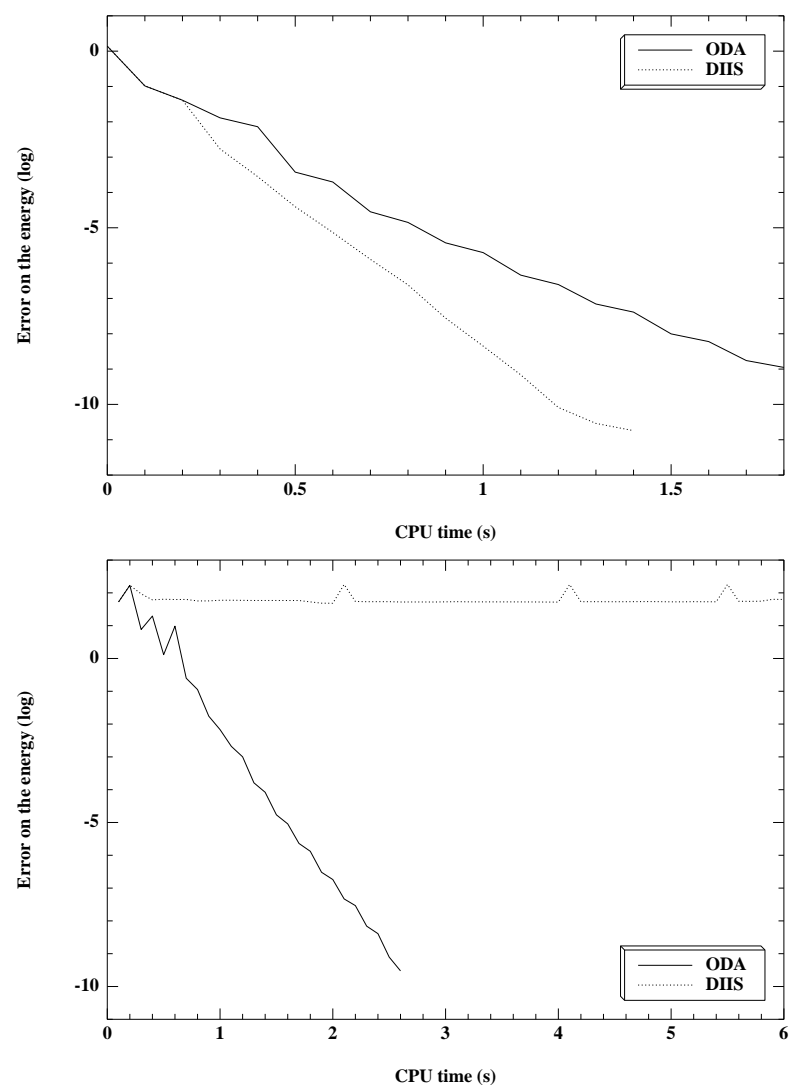

Figure 1: Performances comparées de DIIS et du nouvel algorithme (baptisé ODA pour "Optimal damping algorithm") sur la n-méthyl-2-nitrovinylamine avec deux initial guess calculés de façon différente. 
Cette fois, il ne s'agit plus de (1), mais de l'équation de Schrödinger

$$
\left\{\begin{array}{l}
i \hbar \frac{\partial \psi}{\partial t}=H \psi+E(t) x_{1} \psi, \\
\psi(0)=\psi^{0}
\end{array}\right.
$$

Le problème qu'on étudie est celui du contrôle par laser des évolutions de systèmes chimiques. Le laser se manifeste dans l'équation ci-dessus par le terme $E(t) x_{1} \psi: E(t)$ est son intensité, qui va varier au cours du temps, $x_{1}$ est la coordonnée physique le long de l'axe du laser, $\Psi$ décrit comme toujours l'état du système, lequel, s'il n'était pas soumis au laser, aurait comme Hamiltonien $H$.

Encore une fois, nous ne nous étendrons pas sur la problématique. Nous renvoyons à [22] pour une présentation générale, aussi bien pour les aspects physiques, théoriques ou expérimentaux, que pour les aspects mathématiques théoriques (voir aussi $[9,30]$ pour deux exemples d'études théoriques). Nous préférons détailler un peu les aspects numériques. Le travail est effectué en collaboration avec A. Ben-Haj-Yedder, E. Cancès, M. Pilot, G. Turinici.

Le jeu est d'optimiser $E(t)$ sur un intervalle de temps donné (typiquement quelques femtosecondes $\left.-10^{-15} \mathrm{~s}-\right)$ pour amener le système partant de $\psi_{0}$ au plus près de $\psi_{T}$. En fait, le jeu est bien moindre car poser un tel problème de contrôlabilité optimale dans le cadre où nous sommes n'aurait guère de sens autre que théorique. Il s'agit plutôt d'obtenir à l'instant final, ou au voisinage de l'instant final, une certaine propriété qualitative du système et non un état précis du système (il y a trop d'impondérables dans ce contexte). Comme exemple de cette logique, et sur un cas très simple destiné a terme à devenir plus étoffé, nous examinons donc la question de l'alignement d'une molécule simple. Qui plus est, on ne tient compte que de certains degrés de liberté du système moléculaire, voire d'un seul dans l'étude de base. Le modèle est donc très dégradé par rapport à la sophistication possible d'une équation de Schrödinger dépendante du temps sur un système moléculaire complet. Cependant un tout petit système est déjà suffisant pour rencontrer de grosses diffficultés, et cerner les techniques à employer.

On étudie une molécule triatomique HCN. Dans les coordonnées internes $(\mathbf{R}, \theta, \varphi)$ de la molécule, le Hamiltonien s'écrit

$$
H(\mathbf{R}, \theta, \varphi, \mathbf{t})=\mathbf{T}_{\mathbf{R}}+\mathbf{H}_{\mathbf{r o t}}(\mathbf{R}, \theta, \varphi)+\mathbf{V}(\mathbf{R})+\mathbf{H}_{\text {laser }}(\mathbf{R}, \theta, \varphi, \mathbf{t})
$$

où

$$
H_{r o t}(\mathbf{R}, \theta, \varphi)=-\frac{\hbar^{2}}{\mathbf{2}\left(\mu_{\mathbf{H C N}} \mathbf{R}^{2}+\mu_{\mathbf{C N}} \mathbf{r}^{2}\right)}\left[\frac{1}{\sin \theta} \frac{\partial}{\partial \theta}\left(\sin \theta \frac{\partial}{\partial \theta}\right)+\frac{\mathbf{1}}{\sin ^{2} \theta} \frac{\partial^{2}}{\partial \varphi^{2}}\right]
$$

(22) $H_{\text {laser }}(\mathbf{R}, \theta, \varphi, \mathbf{t})=-\mu_{\mathbf{0}}(\mathbf{R}, \mathbf{r}) \mathbf{E}(\mathbf{t}) \cos \theta-\frac{\mathbf{E}^{2}(\mathbf{t})}{\mathbf{2}}\left[\alpha_{\|}(\mathbf{R}, \mathbf{r}) \cos ^{2} \theta+\alpha_{\perp}(\mathbf{R}, \mathbf{r}) \sin ^{2} \theta\right]$

et $V$ est un potentiel effectif calculé par une méthode $a b$ initio très précise. Le lecteur aura évidemment noté que, contrairement aux modèles des sections précédentes, ce sont les noyaux 


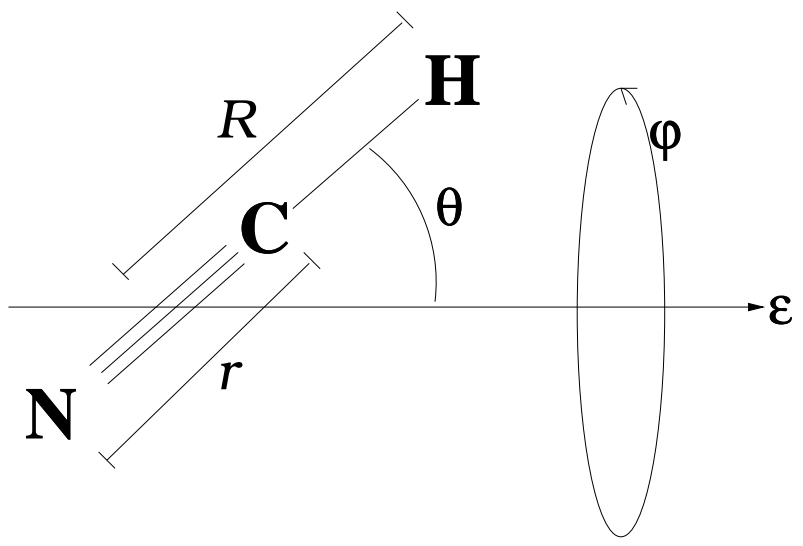

Figure 2: Modèle simplifié pour la molécule $\mathrm{HCN}$ : on cherche à aligner cette molécule linéaire avec le champ laser ici figuré horizontalement.

qui sont ici modélisés comme des objets quantiques et non les électrons. Dans ce domaine, tout dépend de l'application physique.

Pour résoudre (18) avec $H$ donné par (19), le code direct (dû à C. Dion [15]) utilise une technique de splitting d'opérateurs couplée avec une FFT (pour la partie en Laplacien). On utilise 10 lasers (ce qui est actuellement expérimentalement de la science-fiction...), chacun de la forme $E(t)=f(t) E_{0} \cos (w t+\phi)$ où :

$$
f(t)=\left\{\begin{array}{llc}
0 & \text { if } & 0<t<t_{0} \\
\sin ^{2}\left[\frac{t-t_{0}}{t_{1}-t_{0}} \frac{\pi}{2}\right] & \text { if } & t_{0}<t<t_{1} \\
1 & \text { if } & t_{1}<t<t_{2} \\
\sin ^{2}\left[\frac{t-t_{3}}{t_{2}-t_{3}} \frac{\pi}{2}\right] & \text { if } & t_{2}<t<t_{3} \\
0 & \text { if } & t>t_{3}
\end{array}\right.
$$

Comme première étape, on considère seulement un rotateur rigide : la seule variable est $\theta$.

Pour optimiser le champ sur la base d'une fonction critère prenant en compte une mesure de l'alignement de la molécule avec le champ laser, on a choisi de différentier automatique-

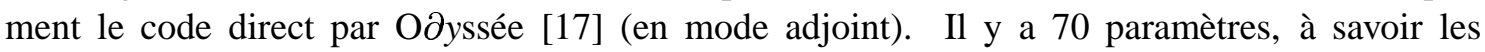
$t_{0}^{i}, t_{1}^{i}, t_{2}^{i}, t_{3}^{i}, E^{i}, w^{i}, \phi^{i}$ pour $i=1,10$. L'algorithme d'optimisation est typiquement un gradient conjugué. On comparera dans un avenir proche [2] les résultats avec ceux obtenus par d'autres types d'algorithmes déterministes et par des algorithmes stochastiques [1]. A court terme, on passera évidemment au cas du Hamiltonien (19) complet, i.e. non réduit à la seule variable $\theta$. Des techniques plus lourdes devront alors être mises en oeuvre. De plus, même sur les cas simples, des techniques liées au contrôle robuste (la notion de robustesse est essentielle dans ce domaine) devront être employées. Une comparaison avec des résultats d'expériences menées en parallèle est prévue. 


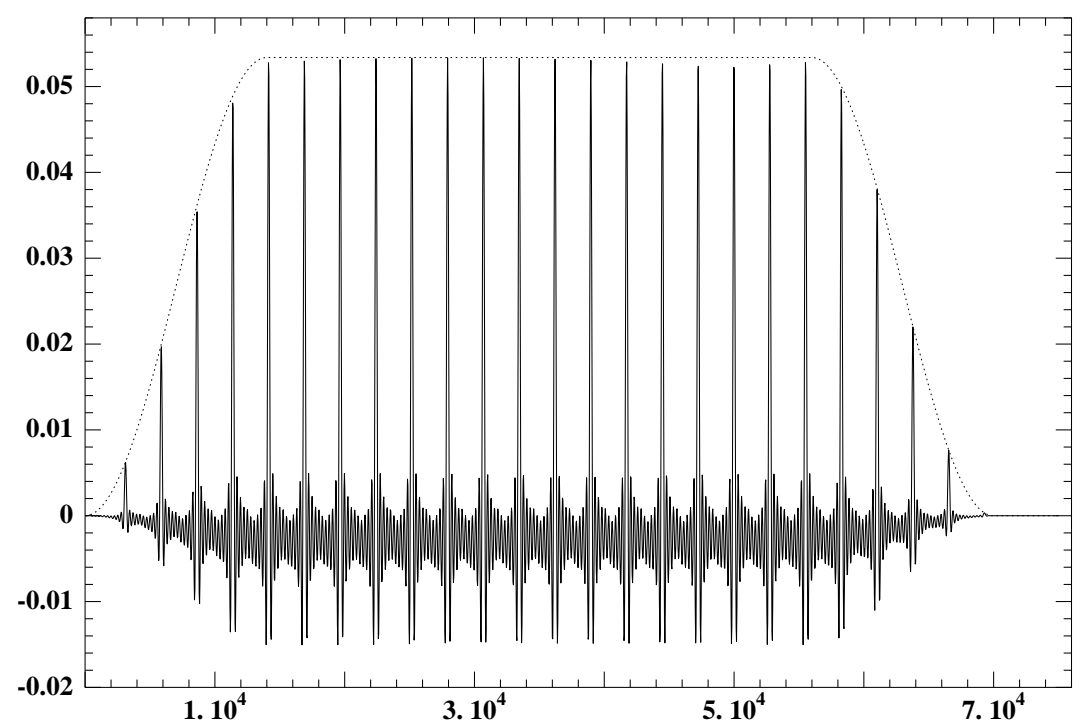

Figure 3: Un champ laser $E(t)$ optimisé.

\section{Perspectives}

Nous n'avons présenté ici que des exemples d'études numériques dans le contexte de la simulation moléculaire. Il s'agit d'un domaine très riche, qui, malgré certaines avancées récentes tout à fait remarquables, reste largement inexploré par les communautés de l'analyse numérique et du calcul scientifique. Beaucoup de travail reste donc à faire.

\section{Remerciements}

Je tiens à remercier l'ensemble des chercheurs participant aux travaux évoqués ici. Outre les collaborateurs "habituels" ( Y. Maday, M. Barrault, A. Ben Haj Yedder, E. Cancès, M. Pilot, G. Turinici), c'est un plaisir de remercier les membres de l'ACI Contrôle des réactions chimiques par laser, supportée par le MENRT : O. Atabek, C. Dion, Th. Horsin, M. Jendoubi, O. Kavian, A. Keller, B. Soep, M. Elhanine, F. Lequéré, J.P. Puel, P. Rouchon. Je tiens aussi à remercier Ch. Faure pour avoir mis O $\partial y$ ssée à notre disposition et I. Charpentier pour ses nombreux conseils.

\section{Références}

[1] M. BARRAUlt \& C. LE BRIS, Optimisation numérique et différentiation automatique pour un problème industriel, Rapport CERMICS 99-179, http://cermics.enpc.fr/reports/CERMICS-99179.ps.gz.

[2] A. BEN-HAJ-YedDER ET AL., travail en préparation. 
[3] P.N. BROWN \& Y. SAAD, Projection methods for solving nonlinear systems of equations., in Nematics. Defects, singularities and patterns in nematic liquid crystals, 1990, NATO ASI Ser., Ser. C 332, 341-355 (1991).

[4] E. CANCÈS, SCF algorithms for Hartree-Fock calculations, in Mathematical models and methods for ab initio Quantum Chemistry, M. Defranceschi \& C. Le Bris Eds., Lecture Notes in Chemistry, Springer, à paraître, http://cermics.enpc.fr/reports/CERMICS-99-184.ps.gz.

[5] E. CANCÈS , M. DefrAnCESCHI, C. LE BRIS, Some recent mathematical contributions to Quantum Chemistry, Int. J. Quant. Chem., 74, 1999, pp 553-557.

[6] E. CANCÈS, C. LE BRIS, On the convergence of SCF algorithms for the Hartree-Fock equations, Math. Model. Num. Anal., à paraître, http://cermics.enpc.fr/ cances/SCF.ps.gz.

[7] E. CANCÈS, C. LE BRIS, Can we outperform the DIIS approach for SCF calculations ?, Int. J. Quant. Chem., Volume 79, Issue 2, 2000, pp 82-90.

[8] E. CANCÈS, C. LE BRIS, en préparation.

[9] E. CAncÈs, C. LE BRIS, M. PILOT, Contrôle optimal bilinéaire sur une équation de Schrödinger, Note aux Comptes Rendus de l'Académie des Sciences, t. 330, Série 1, p 567-571, 2000.

[10] A. DANiEls \& G. SCUSERIA, What is the best alternative to diagonalization of the Hamiltonian in large scale semiempirical calculations ?, J. Chem. Phys, 110, pp 1321-1328, 1999.

[11] M. Defranceschi \& C. Le Bris, Computing a molecule : A mathematical viewpoint., J. Math. Chem., 21, 1997, pp 1-30.

[12] M. Defranceschi \& C. Le Bris, Computing a molecule in its environment : A mathematical viewpoint., Int. J. Quant. Chem.,71, pp 227-250, 1999.

[13] M. Defranceschi \& C. Le BRIs, Eds., Mathematical models and methods for ab initio Quantum Chemistry, Lecture Notes in Chemistry, Springer, volume 74, à paraître, Octobre 2000.

[14] P. Deuflhard ET Al., Eds. Computational molecular dynamics: challenges, methods, ideas, Lecture Notes in Computational Science and Engineering. 4., Springer, 1997.

[15] C. Dion, Dynamique de l'alignement et de l'orientation moléculaire induite par laser. Simulations numériques sur HCN en champ infrarouge. Thèse de l'Université de Sherbrooke et de l'Université de Paris-Sud, 1999.

[16] R.M. Dreizler \& E.K.U. Gross, Density functional theory, Springer-Verlag, 1990.

[17] $\mathrm{CH}$. FAURE Documentation succinte d'Odyssée version 1.6. INRIA, http://www.inria.fr/RRRT/publications-fra.html, décembre 1996.

[18] D. FRENKEL \& B. SMIT, Understanding molecular simulation. From algorithms to applications, Academic Press, 1996.

[19] L. JAY, H. KIM, Y. SAAD, J. CHELIKOWSKI, Electronic structure calculations for plane-wave codes without diagonalization, Computer Physics Communications, Comp. Phys. Comm, 118, pp 21-31, 1999.

[20] S. Goedecker, Linear scaling electronic structure methods, Rev. of Modern Physics, 71, 4, 1999.

[21] W.J. Hehre, L. Radom, P.v.R. Schleyer And J.A. Pople, Ab initio molecular orbital theory, J. Wiley and Sons, 1986 
[22] C. LE BRIS, Control theory applied to Quantum Chemistry: Some tracks, in International Conference on systems governed by PDEs, Nancy, March 1999, ESAIM Proceedings, vol. 8, pp 77-94, 2000, http: //www. emath.fr/Maths/Proc/Vol. 8/ABS/06Lebris/06Lebris.html.

[23] C. LE BRIS, Mathématiques Appliquées et Chimie Quantique, Matapli, Bulletin de la Société de Mathématiques Appliquées et Industrielles, 58, p 25-36, Avril 1999, http://cermics.enpc.fr/ lebris/matapli.pdf.

[24] Y. MAdAY \& G. Turinici, travail en préparation, voir la Thèse de G. Turinici.

[25] T. Mattson, Ed., Parallel computing in computational chemistry, ACS symposium series 592, Oxford.

[26] R. McWenny, Methods of molecular Quantum Mechanics, Academic Press, 1992.

[27] P. PULAY, Convergence acceleration of iterative sequences. The case of SCF iteration., Chem. Phys. Lett., $\underline{73}, 2$ (1980), pp 393-398.

[28] P. PUlay, Improved SCF convergence acceleration, J. Comp. Chem. 3 (1982) pp 556-560.

[29] A. Szabo \& N.S. OstLund, Modern quantum chemistry: an introduction to advanced electronic structure theory, MacMillan, New-York, 1982. 
[30] G. TuRINICI, Contrôlabilité exacte de la population des états propres dans les systèmes quantiques bilinéaires, C.R.Acad. Sci.Paris, t. 330, Serie I, p.327-332, 2000.

[31] W.F. Van Gunsteren et Al., Eds., Computer simulation of Biomolecular systems, volumes 1,2,3, ESCOM, 1989,1993,1997.

Claude Le Bris

\section{CERMICS}

Ecole Nationale des Ponts \& Chaussées

6 et 8 avenue Blaise Pascal

Cité Descartes

Champs-sur-Marne

77455 Marne-La-Vallée Cedex 2, France

mailto:lebris@cermics.enpc.fr

http://cermics.enpc.fr/ lebris/home.html 